\title{
Burnout und Gratifikationskrisen im Längsschnitt bei Ärztinnen und Ärzten während der fachärztlichen Weiterbildung in Deutschland
}

\section{Burnout and Gratification Crises in Female and Male Physicians during Postgraduate Medical Education in Germany - A Longitudinal Study}

Autoren

Rüya Daniela Kocalevent ${ }^{1}$, Hans Pinnschmidt ${ }^{2}$, Sarah Nehls ${ }^{1}$, Sigrid Boczor ${ }^{1}$ (D), Stephanie Siegert ${ }^{1}$, Martin Scherer ${ }^{1}$, Hendrik van den Bussche ${ }^{1}$

Institute

1 Institut und Poliklinik für Allgemeinmedizin, Universitätsklinikum Hamburg-Eppendorf

2 Institut für Medizinische Biometrie und Epidemiologie, Universitätsklinikum Hamburg-Eppendorf

Keywords

Burnout, Ärztliche Weiterbildung, KarMed, Gratifikationskrise

eingereicht 14.01 .2019

akzeptiert 22.11.2019

Bibliografie

DOI https://doi.org/10.1055/a-1068-9843

Online-Publikation: 17.1.2020

Psychother Psych Med 2020; 70: 319-329

(c) Georg Thieme Verlag KG Stuttgart · New York

ISSN 0937-2032

Korrespondenzadresse

Dr. Rüya Daniela Kocalevent

Institut und Poliklinik für Allgemeinmedizin

Universitätsklinikum Hamburg-Eppendorf

Martinistraße 52

20246 Hamburg

r.kocalevent@uke.de

Tab. 7 und Abb. 4 und 5 finden Sie online unter

https://doi.org/10.1055/a-1068-9843.

\section{ZUSAMMENFASSUNG}

Mehrere Studien belegen den hohen beruflichen Stress von Ärzten und Ärztinnen bis hin zu Burnout-Symptomen. Untersucht In der vorliegenden Studie wurde das Belastungserleben von Ärzten und Ärztinnen im längsschnittlichen Verlauf der fachärztlichen Weiterbildung untersucht. Dazu wurde der Frage nachgegangen, wie sich das Burnouterleben unter Berücksichtigung von Gratifikationskrisen von Beginn bis zum Ende der Weiterbildung an innerhalb von 6 Jahren verändert und ob es jeweils einen Einfluss von Geschlecht und Elternstatus gibt. Die Datenerhebung erfolgte im Rahmen der KarMed Studie. Eingesetzt wurden das Maslach Burnout Inventar (MBI-HSS (MP)) und der Effort-Reward-Imbalance Fragebogen (ERI). Die theoretischen Annahmen wurden in allgemeinen linearen Modellen und allgemeinen linearen gemischten Modellen mit Meßwiederholungsdesign statistisch überprüft. Es bestand ein hoher Zusammenhang zwischen dem Ausgangsniveau der Belastung zu Beginn der Weiterbildung und dem Belastungserleben zum Ende der Weiterbildung. Gratifikationskrisen hatten einen signifikanten Einfluss auf die Sub-Skala emotionale Erschöpfung des $\mathrm{MBI}$, nicht aber auf die Subskalen persönliche Leistungsfähigkeit und Depersonalisation. Signifikante Zeiteffekte fanden sich auf allen 3 Sub-Skalen des MBI, mit signifikanten Geschlechtseffekten auf den Subskalen emotionale Erschöpfung und Depersonalistaion. Der Elternstatus weist unter Berücksichtigung der Burnout-Verlaufswerte und Gratifikationskrisen keinen signifikanten Einfluss auf das Burnouterleben auf. Die theoretischen Implikationen und die praktischen Konsequenzen für die Gesundheitsförderung von ÄrztInnen werden ebenso diskutiert wie die Begrenzungen der Untersuchung.

\section{ABSTRACT}

Several studies have demonstrated high work related stress and burnout symptoms in physicians. The study examined the longitudinal relationships of burnout and gratification crisis during 6 years of postgraduate medical education, controlled for gender and parental status. Data acquisition was carried out within the KarMed-study. Administered were the Maslach Burnout Inventory (MBI-HSS (MP)) (MBI) and the Effort-Reward-Imbalance (ERI). The analyses focused on general linear models and general linear mixed models with repeated measure designs. The amount of burnout perceived at the beginning of the postgraduate medical education is highly associated with the perceived amount of burnout at the end. Gratification crisis were significant for one subscale of the MBI (emotional exhaustion), yet not for personal accomplishment nor depersonalization. 
Significant time effects were found on all 3 subscales of the $\mathrm{MBI}$, with gender effects on the subscales emotional exaustion and depersonalization. Having kids did not have significant effects, controlled for all measurement points of burnout as well as for gratification crisis. Theoretical implications and practical consequences for doctors, as well as the limitations of the study, are discussed.

\section{Hintergrund und Zielsetzung}

Dem Beschlussprotokoll des 121. Deutschen Ärztetags im Mai 2018 ist zu entnehmen, dass die Bundesärztekammer sich mit der Gefährdung der (psychischen) Gesundheit von Ärzten ${ }^{1}$ durch die aktuellen Rahmenbedingungen der ärztlichen Tätigkeit befasst. Der Ärztetag hat gefordert, dass die Erhaltung der eigenen psychischen Gesundheit von Medizinstudierenden bzw. Ärzten bereits in die Curricula des Medizinstudiums integriert wird [1]: „Dies beinhaltet die Sensibilisierung für psychische Probleme, deren Erkennung und Prävention sowie die Stärkung der Resilienz im Studium und Arztalltag“ (Top Ic 34).

Mehrere Studien belegen den beruflichen Stress bei Ärzten bis hin zu Burnout-Symptomen [2-8]. Für Deutschland wird angenommen, dass etwa $20 \%$ der Ärzte unter manifesten Symptomen eines Burnout-Syndroms leiden [2,3], was mit einer geringeren Berufszufriedenheit [4] und einer niedrigeren Lebensqualität verbunden ist $[5,6]$. Besonders junge Berufsangehörige scheinen Burnout-gefährdet zu sein [7, 8]. Dabei wird häufig das Maslach Burnout Inventar eingesetzt, das zwischen emotionaler Erschöpfung, Depersonalisierung und subjektiver Leistungsfähigkeit unterscheidet [5].

Ein erhöhtes Stresserleben - im Vergleich zur Allgemeinbevölkerung - findet sich bereits im Medizinstudium [11]. In einer jüngst erschienenen Meta-Analyse von Erschens et al. findet sich eine systematische Übersicht über das Burnouterleben bei Medizinstudierenden [12]. Die Autoren gaben an, dass die Prävalenzraten von Burnout bei Medizinstudierenden stark variieren (zwischen 7 und bis zu 75,2\%), je nach Studie.

Die Zeit während der ärztlichen Weiterbildung ist prägend in Hinblick auf die persönliche Entwicklung in der Rolle als Arzt und die Qualitätssicherung der Patientenversorgung. Viele Ärzte erleben die Weiterbildung als eine stressvolle, überfordernde Zeitspanne. In der alltäglichen Arbeit gehen sie an die Grenzen ihrer Belastbarkeit $[9,10,13]$.

Im Vergleich zu Chefärzten sowie zur medizinischen Leitung berichteten Ärzte in Weiterbildung von signifikant höheren Arbeitsdefiziten durch Stress. 72 \% der Ärzte in Weiterbildung gaben an, mit hoher bzw. zu hoher Arbeitsintensität konfrontiert zu sein. $41 \%$ erfüllten die Kriterien emotionaler Erschöpfung, 34\% erreichten kritische Werte auf einer Skala der Aversion gegenüber Patienten und Patientinnen. Diese Belastungen korrelierten signifikant mit den Stressoren Überforderung durch starken Arbeitsaufwand, subjektive Arbeitsintensität, inadäquate Freizeit und mangelndem Mitspracherecht [14].

In der Langzeitstudie von Baldwin et al., in der schottische Medizinstudierende während des Studiums und während der Weiter-

1 Aufgrund der besseren Lesbarkeit wird in der vorliegenden Arbeit ausschließlich die männliche Form verwendet. Diese bezieht sich auf Personen beiderlei Geschlechts. bildung interviewt wurden, wurden die Faktoren Arbeitsbedingungen, Anzahl der Notfälle sowie der Patiententode als Stressoren, die mit dem Burnouterleben der Ärzte zusammenhängen, benannt [17]. Ziegler et al. stellten im Rahmen der KarMed-Studie über einen Weiterbildungszeitraum von 4 Jahren fest, dass deutliche Diskrepanzen zwischen den realen und den gewünschten Arbeitszeiten der Ärzte bestehen [16].

Zudem scheinen Geschlechtsunterschiede hinsichtlich des Burnouterlebens bei Ärzten zu bestehen. So gelten Ärztinnen als vermehrt Burnout-gefährdet [17]. Als Gründe kommen die möglich Mehrbelastung durch Schwangerschaft und Elternstatus infrage sowie den damit eventuell verbundenen schlechteren Karrierechancen im Vergleich zu den männlichen Kollegen [18].

Im Zusammenhang mit einer Burnoutgefährdung können auch Gratifikationskrisen eine wichtige Rolle spielen [19]. Gemeint ist hier das Verhältnis von Aufwand und Belohnung, und den damit möglichen verbundenen Gratifikationskrisen, wenn auf Leistung keine Anerkennung bzw. Belohnung folgt [20]. Anerkennung bzw. Belohnung kann in Form von Wertschätzung, Gehalt, Sicherheit des Arbeitsplatzes oder beruflichem Aufstieg erfolgen. Diese beiden Komponenten stehen in einer reziproken Beziehung zueinander. Erfolgt nämlich keine Gegenleistung entstehen durch den Belohnungsmangel negative Emotionen, die sich weiter negativ auf die Selbstregulation auswirken können. Man spricht in dem Zusammenhang von Gratifikationskrisen. Der dadurch hervorgerufene Stress steht im Zusammenhang mit somatischen sowie psychischen Erkrankungen, wie Depression, als auch mit Burnout, und Einbußen in der Lebensqualität [21].

Die Ergebnisse der querschnittlichen Studie von Häusler et al. zeigen, dass das Erleben einer Gratifikationskrise mit der Entwicklung einer Burnout-Symptomatik zusammenhängt. Wenn die Balance zwischen Arbeitsaufwand und Belohnung nicht gegeben ist, habe das besonders auf die Berufsgruppe der Ärzte einen großen Einfluss [19].

Daraus ergeben sich für die vorliegende Arbeit folgende Fragestellungen:

- In welchem Ausmaß verändert sich das Burnouterleben von Beginn bis nach 6 Jahren Weiterbildung unter Berücksichtigung von Geschlecht und Elternstatus?

- Welchen Einfluss haben Gratifikationskrisen nach 6 Jahren

Weiterbildung auf das Burnouterleben unter Berücksichtigung von Geschlecht und Elternstatus?“

\section{Methodik}

Die Studie ist Teil des Forschungsvorhabens „Karriereverläufe von Ärzten in der fachärztlichen Weiterbildung“ („KarMed“), der ersten und bisher einzigen multizentrischen prospektiv-längsschnittlichen Studie, die den Karriereverlauf von Ärzten in Deutschland vom Berufseinstieg bis zur Facharztanerkennung untersucht. Die 
Studie wurde von der Ethik-Kommission der Hamburger Ärztekammer genehmigt (PV 3063).

Untersucht wurde die berufliche Entwicklung von 1012 Ärztinnen (665 Frauen) und Ärzten (346 Männer) vom Ende der ärztlichen Ausbildung bis 6 Jahre in ärztlicher Weiterbildung. Dabei handelte es sich um Absolventinnen und Absolventen des Medizinstudiums aus den medizinischen Fakultäten in Erlangen, Gießen, Hamburg, Heidelberg, Köln, Leipzig und Magdeburg. Für die standardisierte postalische Befragung („T1 “) wurden im Jahr 2010 alle Teilnehmenden der ersten Erhebung ca. ein Jahr nach ihrer ersten Befragung („T0“), die gegen Ende des Praktischen Jahres stattfand, angeschrieben. Die Teilnehmenden sandten den Fragebogen mit anonymem Code zur Wiedererkennung im Längsschnitt sowie einen personalisierten Kontaktbogen getrennt vom Fragebogen an das Studienzentrum in Hamburg zurück (Pseudonymisierung). Dieses Verfahren stellt die Anonymität der Befragung sicher und erlaubt eine Aktualisierung der Adressdatenbank über die Erhebungszeitpunkte hinweg. Alle Teilnehmenden erhielten $10 €$ pro ausgefüllten Fragebogen und nahmen außerdem an der Verlosung eines Preises im Wert von $350 €$ teil. Insgesamt folgten nach T1 5 weitere Erhebungszeitpunkte bis 2015.

Um Burnout und Gratifikationskrisen im Längsschnitt bei Ärzten während der fachärztlichen Weiterbildung zu untersuchen, wurden die Zielpersonen zu Beginn der Weiterbildung (T1) und zu weiteren 4 Messzeitpunkten (T2, T3, T4 und T6) im Verlauf von 6 Jahren Weiterbildung gebeten, das Maslach Burnout Inventar (MBI_ HSS (MP)) sowie den Effort-Reward-Imbalance-Fragebogen (ERI) zu Gratifikationskrisen auszufüllen ( $>$ Abb. 1). Zudem wurden die Zielpersonen gebeten, Angaben zu ihrer Person zu machen (soziodemografische Variablen, u. a. Geschlecht); zusätzlich wurde der Elternstatus abgefragt („Leben ein oder mehrere Kinder dauerhaft oder überwiegend in Ihrem Haushalt?").

Des Weiteren wurden die Zielpersonen gebeten, einen Erhebungsbogen, der mehrere Fragebögen enthielt, auszufüllen. Dieser enthielt zu T1, T2, T3, T4 und T6 den Effort-Reward-ImbalanceFragebogen (ERI) zu Gratifikationskrisen sowie das Maslach Burnout Inventar (MBI).

Der Effort-Reward-Imbalance-Fragebogen (ERI) misst das individuell wahrgenommene Verhältnis von Beanspruchung/investierter Aufwand und Belohnung in der Arbeitswelt [22]. Nach dem theo- retischen Modell ist ein Gleichgewicht von Beanspruchung und Belohnung Voraussetzung für Arbeitszufriedenheit und arbeitsplatzbezogene Gesundheit [22]. Die Belohnung kann sowohl in einem angemessenen Entgelt als auch in immateriellen Gratifikationen wie Wertschätzung der Arbeit durch Vorgesetzte und/oder Anerkennung durch Kolleginnen und Kollegen bestehen. Im Falle einer Ausgewogenheit beider Komponenten hat der Quotient Beanspruchung/Belohnung den Wert $\leq 1$. Ein Wert $>1$ wird als berufliche Gratifikationskrise definiert [23, 24].

Das Burnout-Syndrom wird nach Maslach (MBI-HSS (MP)) als ein Syndrom beschrieben, das durch emotionale Erschöpfung (emotional exhaustion, MBI-EE: 9 Items), Depersonalisation (gefühllose, gleichgültige oder zynische Einstellung gegenüber Klienten bzw. Patienten) (depersonalisation, MBI-DP: 5 Items) und eine positive Einschätzung der persönlichen Leistung gekennzeichnet ist (personal accomplishment, MBI-PA: 8 Items) [5]. In einer systematischen Literaturübersicht von Rotenstein et al. wird die Heterogenität der Verwendung des Burnout-Begriffs und den damit assoziierten Cut-off-Werten problematisiert [25]. Die Cut-off-Werte des Maslach Burnout Inventar wurden in der vorliegenden Arbeit einer deutschen Stichprobe von psychosozialen Helferberufen entnommen [28], um möglichst genau der Berufsgruppe und Nationalität entsprechen zu können. Normwerte hinsichtlich niedrigem, mittlerem und hohem Ausprägungsgrad wurden für die 3 Subskalen gebildet, hinsichtlich niedrigem, mittlerem und hohem Ausprägungsgrad: Emotionale Erschöpfung: hoch > = 2,87; Depersonalisation: hoch > = 2,60; Persönliche Leistungsfähigkeit: hoch $>=4,87$ [26].

\section{Datenanalyse}

Alle statistischen Analysen wurden explorativ mit SPSS für Windows Version 25 durchgeführt. Das Signifikanzniveau wurde auf alpha $=0,05$ (2-seitig) festgelegt [27]. Es wurde 2-seitig getestet, da es sich um keine gerichteten Hypothesen handelt. Es sollte getestet werden, ob es Unterschiede bzw. Effekte - unter Berücksichtigung beider Richtungen - gibt. Beschreibende Statistiken sind Mittelwert und Standardabweichung für kontinuierliche Variablen (MBI, ERI) sowie absolute und prozentuale Häufigkeiten für kategoriale Variablen (Geschlecht, Elternstatus). Korrelationen zwischen Variablen kontinuierlicher oder ordinaler Skalierung wurden

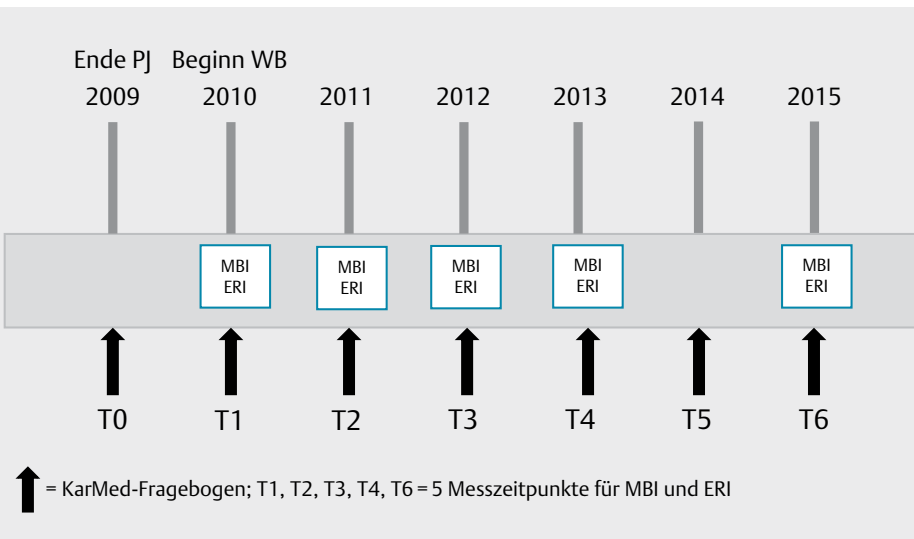

Abb. 1 Messzeitpunkte und Einordnung der Zielvariablen Burnout (gemessen mit dem MBI) und Gratifikationskrisen (gemessen mit dem ERI). 
mittels Spearman-Korrelationsanalysen geprüft. Die SPSS-Routine GENLINMIXED wurde verwandt, um durch allgemeine lineare gemischte Modelle mit Meßwiederholungsdesign zu überprüfen, welche Zusammenhänge es zwischen dem zeitlichen Verlauf von emotionaler Erschöpfung, persönlicher Leistungsfähigkeit und Depersonalisation jeweils zu T6 und den Einflussgrößen Zeit (4 Messzeitpunkte), Geschlecht zu T1, Elternstatus zu T6, emotionaler Erschöpfung, persönlicher Leistungsfähigkeit und Depersonalisation jeweils zu T1 sowie Gratifikationskrisen zu T1 und dem zeitlich aktuellen Zustand von Gratifikationskrisen zu T6 gibt. Für die Identifikationsnummer der Teilnehmer, „ID“, wurde ein zufälliger Effekt angenommen und Zeit innerhalb ID als Messwiederholung. Die Modelle für die 3 Skalen emotionale Erschöpfung, persönliche Leistungsfähigkeit und Depersonalisation wurden jeweils mit (Modell 2) und ohne (Modell 1) die Hinzunahme des Parameters ERI (Gratifikationskrisen) berechnet. Die Modell-berechneten Randmittel und $95 \%$ Konfidenzintervalle wurden für die einzelnen Messzeitpunkte und Kategorien von Geschlecht und Elternstatus dargestellt und mittels paarweiser Kontraste verglichen. Für die festen Effekte wurden jeweils die F-Statistiken und p-Werte der einzelnen Rechenmodelle dargestellt.

\section{Ergebnisse}

\section{Stichprobe}

Die Geschlechterverteilung in der Baselinebefragung betrug $66 \%$ Ärztinnen ( $N=665)$ und $34 \%$ Ärzte $(N=346)$; nach 5-jähriger Weiterbildung ( $=304$ Ärztinnen; N = 126 Ärzte). Die Gesamtstichprobe zu T6 unter Berücksichtigung aller verfügbaren Messzeitpunkte und Kovariaten umfasste N=412 Ärztinnen und Ärzte. Die Rücklaufquoten betrugen, gemessen an den ersten beiden Messzeitpunkten, 85 und $89 \%$. Die Rücklaufquote, gemessen an der Vorjahreserhebung, betrug stets $85 \%$ und mehr, außer in der letzten Befragung (T6), die eine Rücklaufquote von $77 \%$ erbrachte.

Die Drop-out Rate lag zu T6 im Vergleich zur Baselinebefragung im Hinblick auf den ERI und den MBI sowie die Kovariaten Geschlecht und Elternstatus bei $59,25 \%$.

- Tab. 1 gibt einen Überblick über die soziodemografischen Variablen.

Zwischen der Befragung im Praktischen Jahr (T0) und der nach 6 Weiterbildungsjahren (T6) stieg der Frauenanteil in der Kohorte um 4,5 Prozentpunkte. Nach 6-jähriger Weiterbildung betrug der Altersmedian bei den Ärzten 34 Jahre, bei den Ärztinnen 33 Jahre.

> Tab. 1 Soziodemografische Daten zum fachärztlichen Weiterbildungsbeginn $\mathrm{T} 1(\mathrm{~N}=838)$.

\begin{tabular}{|l|l|l|}
\hline & $\boldsymbol{N}(\%)$ & $\boldsymbol{M}$ (SD) \\
\hline Ärztinnen & $564(67,3 \%)$ & - \\
\hline Ärzte & $274(32,7 \%)$ & - \\
\hline Alter & - & $28,2(2,6)$ \\
\hline Kinder & $88(10,5 \%)$ & - \\
\hline Feste Partnerschaft & $608(72,8 \%)$ & - \\
\hline
\end{tabular}

Der Anteil der Befragten mit Kind stieg in den 6 Jahren von $8 \%$ auf $48 \%$, mit einem großen Sprung zu T5 (T4: 29\%, T5: $43 \%$ ).

\section{Korrelative Zusammenhänge zwischen Burnout und Gratifikationserwartungen}

Der höchste korrelative Zusammenhang für die Subskala persönliche Leistungsfähigkeit nach 6 Jahren wies der Messwert der persönlichen Leistungsfähigkeit zu Beginn der Weiterbildung auf $(r=0,46 ; p<0,05)$ (Tab. 7 online). Die Subskala emotionale Erschöpfung nach 6 Jahren war am höchsten mit Depersonalisation nach 6 Jahren assoziiert $(r=0,54 ; p<0,05)$ sowie mit emotionaler Erschöpfung zu Beginn der Weiterbildung $(r=0,44 ; p<0,05)$. Depersonalisation nach 6 Jahren wies zudem einen hohen Zusammenhang auf mit Depersonalisation zum Beginn der Weiterbildung $(r=0,50 ; p<0,05)$.

Gratifikationserwartungen, genauer der Quotient von Beanspruchung und der damit verbundenen Erwartung einer Belohnung, waren am höchsten mit der Subskala emotionale Erschöpfung nach 6 Jahren assoziiert $(r=0,15 ; p<0,05)$ und persönlicher Leistungsfähigkeit zu Beginn der Weiterbildung ( $r=0,18$; $p<0,05)$.

\section{Gratifikationserwartungen}

Die Gratifikationserwartungen nahmen signifikant zu von Beginn der Weiterbildung bis 6 Jahre nach der Weiterbildung. \ Tab. 2 gibt die entsprechenden Mittelwerte (und SD) der berufsbezogenen Belastungen nach Geschlecht oder Elternstatus für den ERI Fragebogen wieder (T1 im Vergleich zu T6). In der Stichprobe fanden sich zu T1 bei 8,9\% (Gesamtwert>1) Hinweise auf eine berufliche Gratifikationskrise. Zu T6 gab es einen Anstieg der Gratifikationskrisen auf 94,5\% (Gesamtwert> 1), davon gut ein Drittel über 1,5 (32,1 \%) und 2,5\% über einem Wert von 2.

\section{Burnout Zeiteffekte ohne Hinzunahme von Gratifikationskrisen zum Weiterbildungsende}

Subskala Emotionale Erschöpfung

Zwischen den einzelnen Messzeitpunkten ergaben sich signifikante Unterschiede zwischen T2 und T3 sowie zwischen T2 und T6 (entspricht Messzeitpunkt 5) (>Abb. 2). Dabei zeigt sich ein Geschlechtseffekt über die Messzeitpunkte hinweg ( $p<0,05)$ (Abb. 4 online). Ärztinnen wiesen im Vergleich zu Ärzten signifikant höhere Werte für emotionale Erschöpfung über die Messzeitpunkte hinweg auf. Der Elternstatus weist unter Berücksichtigung aller Messzeitpunkte des MBI keinen signifikanten Effekt auf.

Der höchste geschätzte Mittelwert für emotionale Erschöpfung über die Messzeitpunkte ( $\triangleright$ Tab. 3a) findet sich zum Messzeitpunkt 4 (entspricht T4) mit $M=3,50(S D=0,26)$. Die Streuung ist zu T4 ebenfalls am größten.

\section{Subskala persönliche Leistungsfähigkeit}

Zwischen den einzelnen Messzeitpunkten ergaben sich signifikante Unterschiede zwischen allen Messzeitpunkten bis auf T3 zu T6 (entspricht Messzeitpunkt 5) ( A Abb. 2). Dabei zeigte sich kein Geschlechtseffekt über die Messzeitpunkte hinweg. Der Elternstatus wies ebenfalls keinen signifikanten Effekt unter Berücksichtigung aller Messzeitpunkte des MBI auf. 


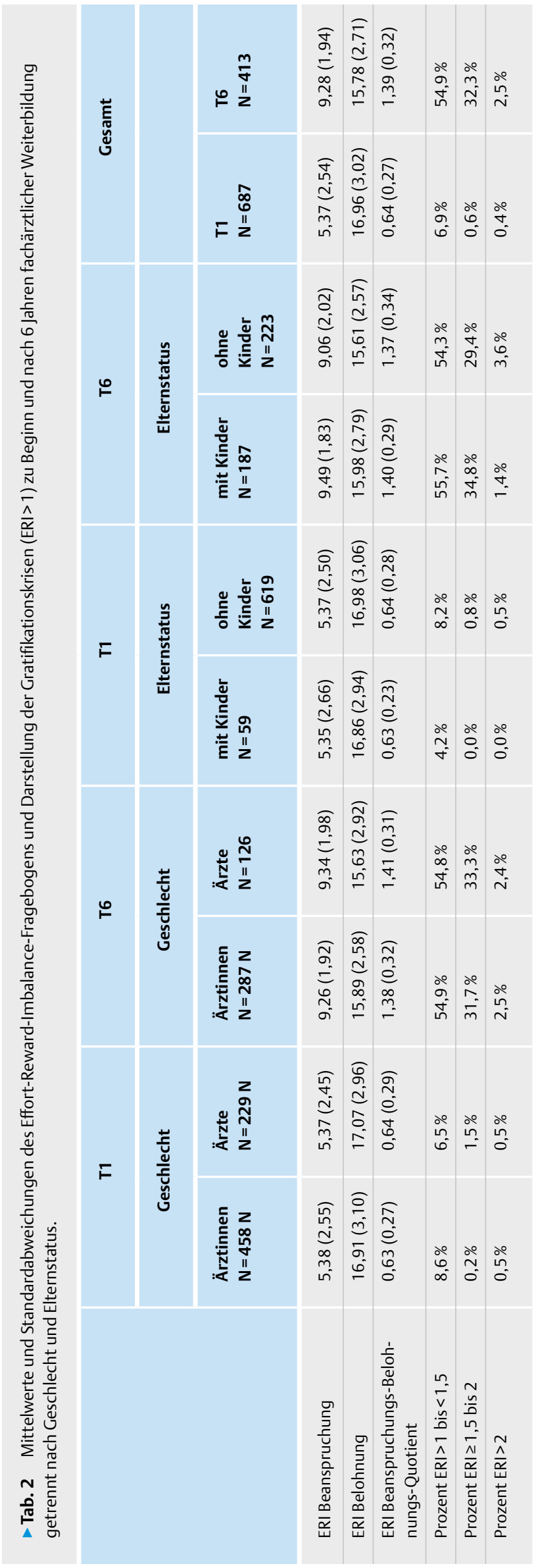

Der höchste geschätzte Mittelwert findet sich zum Messzeitpunkt 5 (entspricht T6) mit M=5,79 (SD =0,003). Die persönliche Leistungsfähigkeit stieg somit kontinuierlich an.

\section{Subskala Depersonalisation}

Zwischen den einzelnen Messzeitpunkten ergaben sich keine signifikanten Unterschiede bis auf T2 zu T3 und T2 zu T5 (> Abb. 2). Dabei zeigte sich ein Geschlechtseffekt über die Messzeitpunkte hinweg $(p<0,05)$. Ärzte wiesen signifikant höhere Werte für Depersonalisation über die Messzeitpunkte hinweg im Vergleich zu Ärztinnen auf. Der Elternstatus wies keinen signifikanten Effekt unter Berücksichtigung aller Messzeitpunkte des MBI auf ( $>$ Tab. 3b).

Der höchste geschätzte Mittelwert fand sich zum Messzeitpunkt 3 (entspricht T3) mit M=3,01 (SD=0,05). Danach sanken die Werte für die Skala Depersonalisation wieder ab ( $\triangleright$ Tab. 3c).

\section{Burnout Zeiteffekte unter Berücksichtigung von Gratifikationskrisen}

Die ( $\triangleright$ Tab. $4 \mathbf{a}-\mathbf{c}$ ) verweisen auf die festen Effekte der einzelnen Einflussgrößen einschließlich der Angabe ihrer p-Werte. Dabei zeigten Gratifikationskrisen nach 6 Weiterbildungsjahren einen signifikanten Effekt auf die emotionale Erschöpfung auf, nicht aber zu Beginn der Weiterbildung. Bei der persönlichen Leistungsfähigkeit verhielt es sich umgekehrt. Hier sehen wir einen signifikanten Effekt der Gratifikationskrisen zu Beginn der Weiterbildung, aber nicht mehr nach 6 Weiterbildungsjahren. Die persönliche Leistungsfähigkeit war am Ende der Weiterbildung nach 6 Weiterbildungsjahren also unbeeinflusst von Gratifikationskrisen. Depersonalisation wies weder zu Beginn noch zum Ende der Weiterbildung einen Effekt durch Gratifikationskrisen auf.

\section{Subskala Emotionale Erschöpfung}

- Tab. 5a gibt die geschätzten Mittelwerte über die Zeit hinweg wieder. Alle geschätzten Mittelwerte sind als „hoch“ einzustufen nach den von Enzmann \& Kleiber ermittelten Normwerten [26]. Der höchste geschätzte Mittelwert findet sich zum Messzeitpunkt 4 (entspricht T4) $\mathrm{M}=4,08$ (SD = 0,05). Zwischen den einzelnen Messzeitpunkten ergaben sich signifikante Unterschiede bis auf die Messzeitpunkte T3 zu T4 ( $\triangleright$ Abb. 3). Dabei zeigte sich ein Geschlechtseffekt über die Messzeitpunkte hinweg $(p<0,05)$ (Abb. 5 online). Ärztinnen wiesen signifikant höhere Werte für emotionale Erschöpfung über die Messzeitpunkte hinweg auf im Vergleich zu Ärzten. Der Elternstatus wies keinen signifikanten Effekt auf.

\section{Subskala persönliche Leistungsfähigkeit}

- Tab. 5b gibt die geschätzten Mittelwerte über die Zeit hinweg wieder. Die persönliche Leistungsfähigkeit nimmt kontinuierlich zu. Bis auf den Messzeitpunkt 2 (entspricht T2) sind die geschätzten Mittelwerte als überdurchschnittlich „hoch“ einzustufen im positiven Sinne [28]. Der höchste geschätzte Mittelwert findet sich zum Messzeitpunkt 5 (entspricht T6) M=5,79 (SD =0,05). Zwischen den einzelnen Messzeitpunkten ergaben sich signifikante Unterschiede bis auf T3 zu T6 (entspricht Messzeitpunkt 5) (\$Abb. 3). Dabei zeigte sich kein Geschlechtseffekt über die Messzeitpunkte hinweg. Der Elternstatus wies ebenfalls keinen signifikanten Effekt auf. 

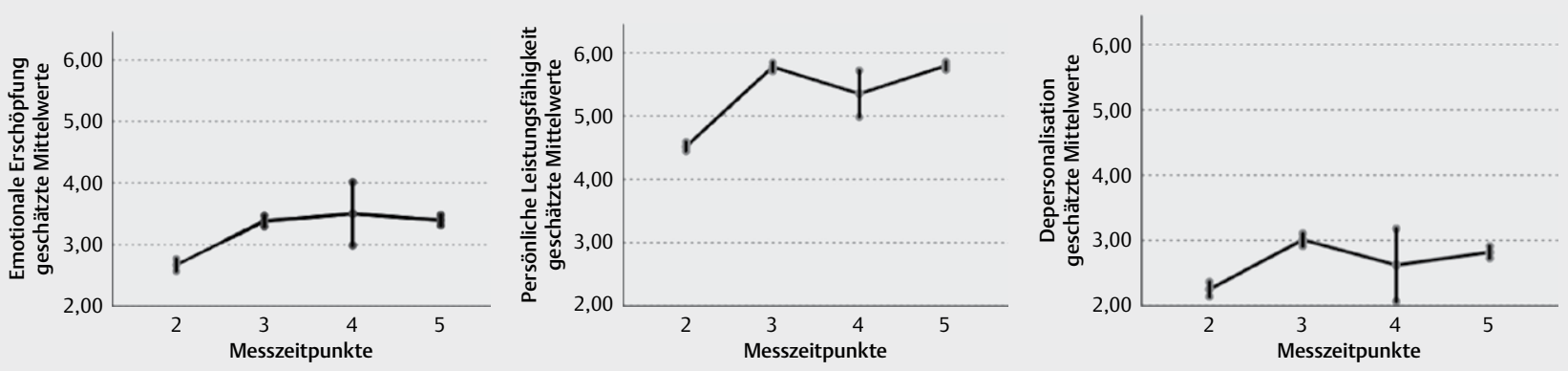

- Abb. 2 Burnouterleben (emotionale Erschöpfung, persönliche Leistungsfähigkeit, Depersonalisation) im zeitlichen Verlauf der ärztlichen Weiterbildung ohne Berücksichtigung von Gratifikationskrisen zum Weiterbildungsende. Stetige Einflussvariablen sind auf folgende Werte festgelegt: Emotionale Erschöpfung zum Messzeitpunkt 1=1,81; Persönliche Leistungsfähigkeit zum Messzeitpunkt 1=3,90; Depersonalisation zum Messzeitpunkt 1 = 1,27; Gratifikationskrisen zum Messzeitpunkt 1 = 0,61; Emotionale Erschöpfung zum Messzeitpunkt 5=3,19 Persönliche Leistungsfähigkeit zum Messzeitpunkt 5=5,36; Depersonalisation zum Messzeitpunkt 5=2,63.

- Tab. 3a Geschätzte Mittelwerte des allgemeinen linearen gemischten Modells mit Meßwiederholungsdesign für die Skala emotionale Erschöpfung des MBI ohne Hinzunahme des ERI (Gratifikationskrisen) nach 6 Jahren Weiterbildung als stetige Einflussvariable *.

\begin{tabular}{|l|l|l|l|l|}
\hline Messzeitpunkte & Mittelwert & SD & $\mathbf{9 5 \%}$ Konfidenzintervall Unterer Wert & 95\% Konfidenzintervall Oberer Wert \\
\hline $\mathbf{5}$ & 3,40 & 0,04 & 3,31 & 3,47 \\
\hline 4 & 3,50 & 0,26 & 2,99 & 4,01 \\
\hline 3 & 3,38 & 0,04 & 3,29 & 3,47 \\
\hline 2 & 2,67 & 0,05 & 2,57 & 2,77 \\
\hline
\end{tabular}

${ }^{*}$ Stetige Einflussvariablen sind auf folgende Werte festgelegt: Emotionale Erschöpfung zum Messzeitpunkt 1=1,81; Persönliche Leistungsfähigkeit zum Messzeitpunkt 1=3,90; Depersonalisation zum Messzeitpunkt 1 =1,27; Gratifikationskrisen zum Messzeitpunkt 1=0,61; Emotionale Erschöpfung zum Messzeitpunkt 5=3,19 Persönliche Leistungsfähigkeit zum Messzeitpunkt 5=5,36; Depersonalisation zum Messzeitpunkt 5=2,63.

> Tab. 3b Geschätzte Mittelwerte des allgemeinen linearen gemischten Modells mit Meßwiederholungsdesign für die Skala persönliche Leistungsfähigkeit des MBI ohne Hinzunahme des ERI (Gratifikationskrisen) nach 6 Jahren Weiterbildung als stetige Einflussvariable *.

\begin{tabular}{|l|l|l|l|l|}
\hline Messzeitpunkte & Mittelwert & SD & $\mathbf{9 5 \%}$ Konfidenzintervall Unterer Wert & 95\% Konfidenzintervall Oberer Wert \\
\hline 5 & 5,79 & 0,03 & 5,73 & 5,86 \\
\hline 4 & 5,35 & 0,18 & 4,98 & 5,72 \\
\hline 3 & 5,78 & 0,04 & 5,71 & 5,84 \\
\hline 2 & 4,51 & 0,04 & 4,44 & 4,59 \\
\hline
\end{tabular}

${ }^{*}$ Stetige Einflussvariablen sind auf folgende Werte festgelegt: Emotionale Erschöpfung zum Messzeitpunkt 1=1,81; Persönliche Leistungsfähigkeit zum Messzeitpunkt 1=3,90; Depersonalisation zum Messzeitpunkt 1=1,27; Gratifikationskrisen zum Messzeitpunkt 1=0,61; Emotionale Erschöpfung zum Messzeitpunkt 5 =3,19 Persönliche Leistungsfähigkeit zum Messzeitpunkt 5=5,36; Depersonalisation zum Messzeitpunkt 5=2,63.

\section{Subskala Depersonalisation}

> Tab. $\mathbf{5 c}$ gibt die geschätzten Mittelwerte über die Zeit hinweg wieder. Die geschätzten Mittelwerte für Depersonalisation liegen für die Messzeitpunkte 2 und 4 im mittleren Bereich - für die Messzeitpunkte 3 und 5 im hohen Bereich [28]. Zwischen den einzelnen Messzeitpunkten ergaben sich keine signifikanten Unterschiede bis auf T2 zu T3 ( $\bullet$ Abb. 3). Dabei zeigte sich ein Geschlechtseffekt über die Messzeitpunkte hinweg $(p<0,05)$. Ärzte wiesen signifikant höhere Werte für Depersonalisation über die Messzeitpunkte hinweg im Vergleich zu Ärztinnen auf. Der Elternstatus weist keinen signifikanten Effekt auf.

Schaut man sich die Differenzen der geschätzten Mittelwerte der Subskala emotionale Erschöpfung an, fällt auf, dass die Werte unter Berücksichtigung der Gratifikationserwartungen durchweg 
> Tab. 3c Geschätzte Mittelwerte des allgemeinen linearen gemischten Modells mit Meßwiederholungsdesign für die Skala Depersonalistaion des MBI ohne Hinzunahme des ERI (Gratifikationskrisen) nach 6 Jahren Weiterbildung als stetige Einflussvariable *.

\begin{tabular}{|l|l|l|l|l|}
\hline Messzeitpunkte & Mittelwert & SD & $\mathbf{9 5 \%}$ Konfidenzintervall Unterer Wert & $\mathbf{9 5 \% ~ K o n f i d e n z i n t e r v a l l ~ O b e r e r ~ W e r t ~}$ \\
\hline 5 & 2,82 & 0,05 & 2,73 & 2,90 \\
\hline 4 & 2,62 & 0,28 & 2,06 & 3,18 \\
\hline 3 & 3,01 & 0,05 & 2,91 & 3,10 \\
\hline 2 & 2,25 & 0,06 & 2,13 & 2,36 \\
\hline
\end{tabular}

* Stetige Einflussvariablen sind auf folgende Werte festgelegt: Emotionale Erschöpfung zum Messzeitpunkt 1=1,81; Persönliche Leistungsfähigkeit zum Messzeitpunkt 1 = 3,90; Depersonalisation zum Messzeitpunkt 1=1,27; Gratifikationskrisen zum Messzeitpunkt 1=0,61; Emotionale Erschöpfung zum Messzeitpunkt 5 =3,19 Persönliche Leistungsfähigkeit zum Messzeitpunkt 5 =5,36; Depersonalisation zum Messzeitpunkt 5=2,63.

- Tab. 4a Feste Effekte der einzelnen Einflusgrößen des allgemeinen linearen gemischten Modells auf die Skala emotionale Erschöpfung des MBI nach 6 Jahren Weiterbildung, einschließlich der p-Werte.

\begin{tabular}{|c|c|c|c|c|}
\hline & F-Wert & $d f 1$ & $d f 2$ & Signifikanz \\
\hline Korrigiertes Modell & 142,80 & 12 & 1119 & 0,000 \\
\hline Zeit & 53,92 & 3 & 1119 & 0,000 \\
\hline Geschlecht & 5,88 & 1 & 1119 & 0,015 \\
\hline Elternstatus & 1,46 & 1 & 1119 & 0,227 \\
\hline Emotionale Erschöpfung T1 & 209,88 & 1 & 1119 & 0,000 \\
\hline Persönl. Leistungsf. T1 & 0,415 & 1 & 1119 & 0,519 \\
\hline Depersonalisation T1 & 52,74 & 1 & 1119 & 0,000 \\
\hline Gratifikationserwartungen T1 & 0,56 & 1 & 1119 & 0,455 \\
\hline Persönl. Leistungsf. T6 & 31,07 & 1 & 1119 & 0,000 \\
\hline Depersonalisation zu T6 & 285,45 & 1 & 1119 & 0,000 \\
\hline Gratifikationserwartungen T6 & 91,58 & 1 & 1119 & 0,000 \\
\hline
\end{tabular}

> Tab. 4b Feste Effekte der einzelnen Einflusgrößen des allgemeinen linearen gemischten Modells auf die Skala persönliche Leistungsfähigkeit des MBI nach 6 Jahren Weiterbildung, einschließlich der p-Werte.

\begin{tabular}{|c|c|c|c|c|}
\hline & $\boldsymbol{F}$ & $d f 1$ & $d f 2$ & Signifikanz \\
\hline Korrigiertes Modell & 142,83 & 12 & 1119 & 0,000 \\
\hline Zeit & 395,35 & 3 & 1119 & 0,000 \\
\hline Geschlecht & 1,10 & 1 & 1119 & 0,295 \\
\hline Elternstatus & 1,30 & 1 & 1119 & 0,255 \\
\hline Emotionale Erschöpfung T1 & 8,25 & 1 & 1119 & 0,004 \\
\hline Persönl. Leistungsf. T1 & 156,05 & 1 & 1119 & 0,000 \\
\hline Depersonalisation $\mathrm{T} 1$ & 0,02 & 1 & 1119 & 0,878 \\
\hline Gratifikationserwartungen T1 & 17,48 & 1 & 1119 & 0,000 \\
\hline Emotionale Erschöpfung T6 & 30,36 & 1 & 1119 & 0,000 \\
\hline Depersonalisation zu T6 & 22,90 & 1 & 1119 & 0,000 \\
\hline Gratifikationserwartungen T6 & 0,05 & 1 & 1119 & 0,822 \\
\hline
\end{tabular}


- Tab. 4c Feste Effekte der einzelnen Einflusgrößen des allgemeinen linearen gemischten Modells auf die Skala Depersonalisation des MBI nach 6 Jahren Weiterbildung, einschließlich der p-Werte.

\begin{tabular}{|c|c|c|c|c|}
\hline & $\boldsymbol{F}$ & $d f 1$ & $d f 2$ & Signifikanz \\
\hline Korrigiertes Modell & 125,20 & 12 & 1119 & 0,000 \\
\hline Zeit & 37,25 & 3 & 1119 & 0,000 \\
\hline Geschlecht & 21,54 & 1 & 1119 & 0,000 \\
\hline Elternstatus & 0,01 & 1 & 1119 & 0,924 \\
\hline Emotionale Erschöpfung T1 & 81,45 & 1 & 1119 & 0,000 \\
\hline Persönl. Leistungsf. T1 & 6,90 & 1 & 1119 & 0,009 \\
\hline Depersonalisation T1 & 261,03 & 1 & 1119 & 0,000 \\
\hline Gratifikationserwartungen T1 & 0,35 & 1 & 1119 & 0,554 \\
\hline Emotionale Erschöpfung T6 & 285,09 & 1 & 1119 & 0,000 \\
\hline Persönl. Leistungsf. T6 & 23,21 & 1 & 1119 & 0,000 \\
\hline Gratifikationserwartungen T6 & 0,39 & 1 & 1119 & 0,531 \\
\hline
\end{tabular}

> Tab. 5a Geschätzte Mittelwerte des allgemeinen linearen gemischten Modells mit Meßwiederholungsdesign für die Skala emotionale Erschöpfung des MBI unter Hinzunahme des ERI (Gratifikationskrisen) nach 6 Jahren Weiterbildung als stetige Einflussvariable *.

\begin{tabular}{|l|l|l|l|l|}
\hline Messzeitpunkte & Mittelwert & SD & $\begin{array}{l}\text { 95\% Konfidenzintervall } \\
\text { Unterer Wert }\end{array}$ & $\begin{array}{l}\text { 95\% Konfidenzintervall } \\
\text { Oberer Wert }\end{array}$ \\
\hline 5 & 3,05 & 0,05 & 2,95 & 3,16 \\
\hline 4 & 4,08 & 0,26 & 3,57 & 4,59 \\
\hline 3 & 3,58 & 0,05 & 3,48 & 3,67 \\
\hline 2 & 2,86 & 0,05 & 2,76 & 2,96 \\
\hline
\end{tabular}

Emotionale Erschöpfung zum Messzeitpunkt 1=1,81; Persönliche Leistungsfähigkeit zum Messzeitpunkt 1=3,90; Depersonalisation zum Messzeitpunkt 1=1,27; Gratifikationskrisen zum Messzeitpunkt 1=0,61; Emotionale Erschöpfung zum Messzeitpunkt 5=3,19 Persönliche Leistungsfähigkeit zum Messzeitpunkt 5=5,36; Depersonalisation zum Messzeitpunkt 5 =2,63; Gratifikationskrisen zum Messzeitpunkt 5=0,91.

> Tab. 5b Geschätzte Mittelwerte des allgemeinen linearen gemischten Modells mit Meßwiederholungsdesign für die Skala persönliche Leistungsfähigkeit des MBI unter Hinzunahme des ERI (Gratifikationskrisen) nach 6 Jahren Weiterbildung als stetige Einflussvariable *.

\begin{tabular}{|c|c|c|c|c|}
\hline Messzeitpunkte & Mittelwert & SD & $\begin{array}{l}\text { 95\% Konfidenzintervall } \\
\text { Unterer Wert }\end{array}$ & $\begin{array}{l}95 \% \text { Konfidenzintervall } \\
\text { Oberer Wert }\end{array}$ \\
\hline 5 & 5,79 & 0,05 & 5,70 & 5,88 \\
\hline 4 & 5,36 & 0,20 & 4,98 & 5,74 \\
\hline 3 & 5,78 & 0,04 & 5,70 & 5,86 \\
\hline 2 & 4,52 & 0,04 & 4,44 & 4,60 \\
\hline
\end{tabular}

Emotionale Erschöpfung zum Messzeitpunkt 1=1,81; Persönliche Leistungsfähigkeit zum Messzeitpunkt 1=3,90; Depersonalisation zum Messzeitpunkt 1 =1,27; Gratifikationskrisen zum Messzeitpunkt 1=0,61; Emotionale Erschöpfung zum Messzeitpunkt 5=3,19 Persönliche Leistungsfähigkeit zum Messzeitpunkt 5 =5,36; Depersonalisation zum Messzeitpunkt 5=2,63; Gratifikationskrisen zum Messzeitpunkt 5=0,91.

höher ausfallen - bis auf den letzten Messzeitpunkt (T6 entspricht Messzeitpunkt 5). Die größte Differenz findet sich zum Messzeitpunkt $4(+0,57)(\triangleright$ Tab. 6$)$.

\section{Diskussion}

Es wurden deutsche Ärzte längsschnittlich im Verlauf der Weiterbildung zu Burnouterleben und Gratifikationskrisen befragt. Das Burnouterleben stieg im zeitlichen Verlauf der fachärztlichen Wei- 
> Tab. 5c Geschätzte Mittelwerte des allgemeinen linearen gemischten Modells mit Meßwiederholungsdesign für die Skala Depersonalisation des MBI unter Hinzunahme des ERI (Gratifikationskrisen) nach 6 Jahren Weiterbildung als stetige Einflussvariable * .

\begin{tabular}{|l|l|l|l|l|}
\hline Messzeitpunkte & Mittelwert & SD & $\begin{array}{l}\mathbf{9 5} \text { Konfidenzintervall } \\
\text { Unterer Wert }\end{array}$ & $\begin{array}{l}\mathbf{9 5} \text { Konfidenzintervall } \\
\text { Oberer Wert }\end{array}$ \\
\hline 5 & 2,84 & 0,06 & 2,72 & 2,96 \\
\hline 4 & 2,58 & 0,30 & 1,99 & 3,15 \\
\hline 3 & 2,99 & 0,05 & 2,89 & 3,10 \\
\hline 2 & 2,24 & 0,06 & 2,12 & 2,36 \\
\end{tabular}

Emotionale Erschöpfung zum Messzeitpunkt 1=1,81; Persönliche Leistungsfähigkeit zum Messzeitpunkt 1=3,90; Depersonalisation zum Messzeitpunkt 1 = 1,27; Gratifikationskrisen zum Messzeitpunkt 1=0,61; Emotionale Erschöpfung zum Messzeitpunkt 5=3,19 Persönliche Leistungsfähigkeit zum Messzeitpunkt 5 =5,36; Depersonalisation zum Messzeitpunkt 5=2,63; Gratifikationskrisen zum Messzeitpunkt 5=0,91.
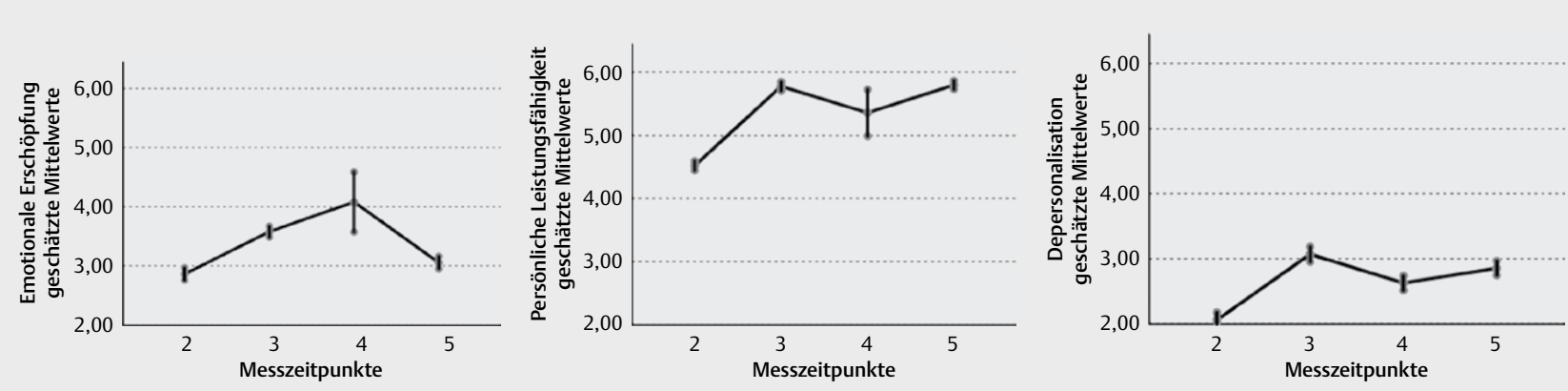

A Abb. 3 Burnouterleben (emotionale Erschöpfung, persönliche Leistungsfähigkeit, Depersonalisation) im zeitlichen Verlauf der ärztlichen Weiterbildung unter Hinzunahme der Einflussvariable Gratifikationskrisen zum Weiterbildungsende. Stetige Einflussvariablen sind auf folgende Werte festgelegt: Emotionale Erschöpfung zum Messzeitpunkt 1=1,81; Persönliche Leistungsfähigkeit zum Messzeitpunkt 1=3,90; Depersonalisation zum Messzeitpunkt 1 = 1.27; Gratifikationskrisen zum Messzeitpunkt 1 =0,61; Emotionale Erschöpfung zum Messzeitpunkt $5=3.19$ Persönliche Leistungsfähigkeit zum Messzeitpunkt 5 =5,36; Depersonalisation zum Messzeitpunkt 5=2.63; Gratifikationskrisen zum Messzeitpunkt $5=0,91$.

> Tab. 6 Geschätze Mittelwerte des allgemeinen linearen gemischten Modells und deren Differenz für die Skala emotionale Erschöpfung des MBI und Darstellung der Konfidenzintervalle (95\%) stratifiziert nach Messzeitpunkten.

\begin{tabular}{|l|l|l|l|l|}
\hline & Messzeitpunkt 2 & Messzeitpunkt 3 & Messzeitpunkt 4 & Messzeitpunkt 5 \\
\hline $\begin{array}{l}\text { Modell 1 (mit Verlaufswerten des } \\
\text { MBI, ohne ERI, vgl. - Abb. 2) }\end{array}$ & $2,68(2,58-2,78)$ & $3,38(3,29-3,46)$ & $3,51(2,99-4,03)$ & $3,39(3,31-3,48)$ \\
\hline $\begin{array}{l}\text { Modell 2 (mit Verlaufswerten des } \\
\text { MBI, mit ERI, vgl. - Abb. 3) }\end{array}$ & $2,86(2,76-2,94)$ & $3,58(3,48-3,67)$ & $4,08(3,57-4,59)$ & $3,05(2,95-3,16)$ \\
\hline Differenz & $\mathbf{+ 0 , 1 8}$ & $\mathbf{+ 0 , 2 0}$ & $\mathbf{+ 0 , 5 7}$ & $-\mathbf{0 , 3 4}$ \\
\hline
\end{tabular}

terbildung an und war zum Ende der 6-jährigen Beobachtungsphase leicht rückläufig. Gratifikationskrisen verstärken diesen Verlauf, insbesondere in Bezug auf emotionale Erschöpfung und Depersonalisation. Zudem reduzieren sich unter Hinzunahme der Gratifikationskrisen die signifikanten Unterschiede der Messzeitpunkte für Depersonalisation auf T2 zu T3. Beide Subskalen, emotionale Erschöpfung als auch Depersonalisation, weisen unter Berücksichtigung der Burnout-Verlaufswerte und der Gratifikationskrisen einen signifikanten Geschlechtseffekt auf. Dabei zeigten sich Ärz- tinnen emotional erschöpfter als Ärzte, während Ärzte höhere Werte von Depersonalisation aufwiesen als Ärztinnen. Die persönliche Leistungsfähigkeit ist hoch und bleibt unbeeinflusst von Gratifikationskrisen nach 6 Weiterbildungsjahren. Denkbar ist hier eine Puffer-Funktion, im Siegrist'schen Sinne intrinsische Faktoren, sogenannte persönliche Bewältigungsstrategien, wie etwa Selbstwirksamkeit, die im Hinblick auf die ansteigende emotionale Erschöpfung und das ansteigende Depersonalisationserleben kompensatorisch wirken [21]. 
Am stärksten war also die Skala emotionale Erschöpfung von Gratifikationskrisen beeinflusst und das insbesondere zu T4. Hier fanden sich die höchsten geschätzten Mittelwerte für emotionale Erschöpfung sowie die größte Streuung. Denkbar wäre, dass Ärztinnen mit Kind die Sub-Gruppe darstellen, die sich am belastetsten zeigt. Hinweise hierfür finden sich, wenn man die Berufsverlaufszufriedenheit zu T4 berücksichtigt [13]. Römer et al. konnten zeigen, dass die Gruppe der Ärztinnen mit Kind im Vergleich zum Rest der Stichprobe zu T4 die geringste Berufsverlaufszufriedenheit aufweist [13].

Es fand sich kein Zeiteffekt über die 5 Messzeitpunkte für den Elternstatus. Geschlechtsspezifische Unterschiede fanden sich im Zusammenhang mit Depersonalisationswerten, bei denen Ärzte häufiger betroffen sind, und emotionaler Erschöpfung, bei denen Ärztinnen häufiger betroffen sind. Diese Effekte zeigten sich stabil über alle 5 Messzeitpunkte hinweg. Es fand sich zudem eine deutliche Steigerung der Häufigkeitsraten von Gratifikationskrisen von $8,9 \%$ zu Beginn auf $94,5 \%$ bis nach 6 Weiterbildungsjahren. In einer Schweizer Studie von [19] wurden die verschiedenen Berufsgruppen der Krankenpfleger und Krankenpflegerinnen, administratives Personal, akademisches Personal und Ärzte querschnittlich miteinander verglichen und nach Symptomen des Burnouts befragt. Ärzte wiesen die höchsten Ausprägungen auf. Wenn die Balance zwischen Arbeitsaufwand und Belohnung nicht gegeben ist, habe das besonders auf die Berufsgruppe der Ärzte einen großen Einfluss [19]. Hier zeigte sich über alle Berufsgruppen hinweg, dass der Konflikt zwischen Arbeit und Privatleben sowie das Erleben einer Gratifikationskrise Prädiktoren für das Entwickeln einer Burnout-Symptomatik sind.

Die Zeit während der Weiterbildung ist nicht nur strukturelle Bedingung im Lebenslauf eines Arztes, sondern auch prägend in Hinblick auf die persönliche Entwicklung in der Rolle als Arzt und die Qualitätssicherung der Patientenversorgung. Während das Leben anderer Menschen bereits von ihnen abhängt, sind sie selbst noch im Lernvorgang und erleben einen exponentiellen Anstieg ihres praktischen Wissens. Die Mischung aus hoher Verantwortung und wenig Kontrollerleben aufgrund noch nicht ausgebildeter Kompetenzen begünstigt die Entwicklung von Burnout [28]. Weiterhin stellte Thomas in ihrer Übersichtsarbeit fest, dass die Ärzte in Weiterbildung über verschiedene Studien hinweg hohe Burnout-Ausprägungen sowie ernsthafte Stressreaktionen erleben und dass Burnout mit schlechter psychischer Gesundheit und gesunkener Arbeitsperformanz assoziiert ist [28]. Da gesunkene Arbeitsperformanz im Arztberuf bedeuten kann, dass gehäuft Fehler vorkommen und dass Patienten und Patientinnen nach Einschätzung der Ärzte in Weiterbildung suboptimal versorgt werden [7], ist es möglicherweise weniger die Gesundheit der Ärzte, als mehr die Gesundheit der Patienten und Patientinnen, die durch die Entwicklung von Burnout-Symptomen gefährdet sein könnte.

Die Grenzen der vorliegenden Studie liegen zum einen in der Stichprobe (Drop-out Rate, fehlende Werte), die repräsentative epidemiologische Angaben in den Subgruppen nicht möglich machte. Zudem lagen neben subjektiven Angaben keine Daten zu objektivierbaren Angaben des Gesundheitszustands vor, sodass Zusammenhänge zwischen Burnout und gesundheitlichen (auch somatischen) Outcomes nicht Gegenstand dieser Studie sein konnten. An dieser Stelle soll die
Notwendigkeit repräsentativer längsschnittlicher Erhebungen unterstrichen werden, um weiter genaueren Aufschluss über die Ursachen und Folgen von stressinduzierten Prozessen und den damit verbundenen Folgen für das Inanspruchnahme- und Gesundheitsverhalten von Ärzten gewinnen zu können.

\section{FAZIT FÜR DIE PRAXIS}

Das Burnouterleben zu Beginn der ärztlichen Weiterbildung bestimmt das Ausmaß der erlebten Belastung nach 6-jähriger Weiterbildung. In Abhängigkeit vom Geschlecht kann es zu einer Verstärkung des Zusammenhangs von Gratifikationskrisen und Burnout, speziell emotionale Erschöpfung, kommen. Zusätzlich fanden sich in unserer Untersuchung Hinweise, dass die persönliche Leistungsfähigkeit im Verlauf der Weiterbildung zunimmt und als Puffer fungiert, der das Burnouterleben der Ärzte möglicherweise kompensiert.

\section{Interessenkonflikt}

Die Autorinnen/Autoren geben an, dass kein Interessenkonflikt besteht.

Literatur

[1] Fuchs C, Koch T, Scriba PC. Perspektiven junger Ärztinnen und Ärzte in der Patientenversorgung. Report Versorgungsforschung Band 4. Köln: Deutscher Ärzteverlag; 2012

[2] Prins JT vdHF, Hoekstra-Weebers JE, Bakker AB et al. Burnout, engagement and resident physicians' self-reported errors. Psychol Health Med 2009; 14: 654-666

[3] Santen SA, Holt DB, Kemp JD et al. Burnout in medical students: examining the prevalence and associated factors. South Med J 2010; 103: $758-763$

[4] Klein J, Grosse FK, Blum K et al. Psychosocial stress at work and perceived quality of care among clinicians in surgery. BMC Health Serv Res 2011; 11: 109

[5] Maslach C, Jackson SE. Maslach Burnout Inventory. Manual $2^{\text {nd }}$ ed. Palo Alto CA: Consulting Psychologists Press; 1986

[6] Köhl-Hackert N, Schultz J-H, Nikendei C et al. Belastet in den Beruf Empathie und Burnout bei Medizinstudierenden am Ende des Praktischen Jahres. Zeitschrift für Evidenz, Fortbildung und Qualität im Gesundheitswesen 2012; 106: 116-124

[7] Shanafelt TD, Bradley KA, Wipf JE et al. Burnout and self-reported patient care in an internal medicine residency program. Ann Intern Med 2002; 136: 358-367

[8] Thomas MR, Dyrbye LN, Huntington JL et al. How do distress and well-being relate to medical student empathy? A multicenter study. J Gen Intern Med 2007; 22: 177-183

[9] Angerer P, Petru R, Weigl M et al. Arbeitsbedingungen und Befinden von Ärzten in der Weiterbildung: Ergebnisse einer Kohortenstudie. In: Schwartz FW \& Angerer P, Hrsg. Arbeitsbedingungen und Befinden von Ärztinnen und Ärzten. Report Versorgungsforschung. Köln: Deutscher Ärzte Verlag 2010: 175-184 
[10] Wegner R, Kostova PT. Belastung und Beanspruchung von Krankenhausärzten zwischen 1975 und 2007. In: Schwartz FW \& Angerer P, Hrsg. Arbeitsbedingungen und Befinden von Ärztinnen und Ärzten. Report Versorgungsforschung. Köln: Deutscher Ärzte Verlag 2010: 243-251

[11] Heinen I, Bullinger M, Kocalevent R. Perceived stress in first year medical students - associations with personal resources and emotional distress. BMC Medical Education 2017; 17: 4

[12] Erschens R, Keifenheim KE, Herrmann-Werner A et al. Professional burnout among medical students: Systematic literature review and meta-analysis. Med Teach 2019; 41: 172-183

[13] Römer R, Ziegler S, Scherer M et al. Berufsverlaufszufriedenheit von Assistenzärzten und -ärztinnen nach vierjähriger Weiterbildung Zeitschrift für Evidenz Fortbildung und Qualität im Gesundheitswesen 2017; 120: 47-53

[14] Biaggi P, Peter S, Ulrich E. Stressors, emotional exhaustion and aversion to patients in residents and chief residents - what can be done? Swiss Med Wkly 2003; 133: 339-346

[15] Baldwin PJ, Dodd M, Wrate W. Young doctors' health - How do working conditions affect attitudes, health and performance? Soc Sci Med 1997; 45: 35-40

[16] Ziegler S, Krause-Solberg L, Scherer M et al. Working hour preferences of female and male residents : Developments over 4 years of postgraduate medical training in Germany. Bundesgesundheitsblatt Gesundheitsforschung Gesundheitsschutz 2017; 60: 1115-1123

[17] Bergner T. Lebensaufgabe statt Lebens-Aufgabe. Dtsch Ärztebl 2004; 101: 2232-2234

[18] Hofmeister D, Rothe K, Alfermann D et al. Ärztemangel selbstgemacht! Über berufliche Belastungen, Gratifikationskrisen und das Geschlechterverhältnis von Berufsanfängern in der Medizin. In: Schwartz FW \& Angerer P, Hrsg. Arbeitsbedingungen und Befinden von Ärztinnen und Ärzten. Report Versorgungsforschung. Köln: Deutscher Ärzte Verlag 2010: 159-174
[19] Häusler N, Bopp M, Hämmig O. Effort-reward Imbalance, Work-privacy Conflict and Burnout Among Hospital Employees. J Occup Environ Med 2018; 60: 183-187. doi:10.1097/JOM.0000000000001287

[20] Siegrist ]. Adverse health effects of high-effort/low-reward conditions. J Occup Health Psychol 1996; 1: 27-41

[21] Siegrist ]. Berufliche Gratifikationskrisen und depressive Störungen. Psychotherapeut 2011; 56: 21-25

[22] Siegrist J, Wege N, Pühlhofer F et al. A short generic measure of work stress in the era of globalization: effort-reward imbalance. Int Arch Occup Environ Health 2009; 82: 1005-1013

[23] Siegrist J, Starke D, Chandola T et al. The measurement of effortreward imbalance at work: European comparisons. Soc Sci Med 2004; 58: 1483-1499

[24] van Vegchela N, de Jongea J, Bosmab $\mathrm{H}$ et al. Reviewing the effortreward imbalance model: drawing up the balance of 45 empirical studies. Soc Sci Med 2005; 60: 1117-1131

[25] Rotenstein L, Torre M, Ramos M et al. Prevalence of Burnout among Physicians. JAMA 2018; 320: 1131-1150

[26] Enzmann D, Kleiber D. Helferleiden Stress und Burnout in Psychosozialen Berufen. Heidelberg: Asanger; 1989

[27] Bortz ], Schuster C. Statistik für Human- und Sozialwissenschaftler. Berlin, Heidelberg: Springer; 2005

[28] Thomas NK. Resident burnout. JAMA 2004; 292: 2913-2915Kocalevent RD et al. Burnout und Gratifikationskrisen im Längsschnitt... Psychother Psych Med 